\title{
La pierre et le béton armé. Note sur Claude Simon et l'architecture moderne
}

Joëlle Gleize

\section{OpenEdition}

1 Journals

Édition électronique

URL : http://journals.openedition.org/ccs/894

DOI : $10.4000 /$ ccs. 894

ISSN : 2558-782X

Éditeur :

Presses universitaires de Rennes, Association des lecteurs de Claude Simon

\section{Édition imprimée}

Date de publication : 31 mai 2014

Pagination : $31-42$

ISBN : 9782753533387

ISSN : $1774-9425$

\section{Référence électronique}

Joëlle Gleize, «La pierre et le béton armé. Note sur Claude Simon et l'architecture moderne », Cahiers Claude Simon [En ligne], 9 | 2014, mis en ligne le 22 septembre 2017, consulté le 21 avril 2019. URL : http://journals.openedition.org/ccs/894 ; DOI : 10.4000/ccs.894 


\title{
LA PIERRE ET LE BÉTON ARMÉ NOTE SUR CLAUDE SIMON ET L'ARCHITECTURE MODERNE
}

\author{
Joëlle GLEIZE \\ Université d'Aix-Marseille
}

Comme Balzac et Proust, Claude Simon emploie la métaphore architecturale pour parler de la composition d'une ouvre littéraire: ainsi, à propos de La Route des Flandres, il dit avoir voulu " forger une structure " "qui [lui] permette de présenter les uns après les autres des éléments qui dans la réalité se superposent, de retrouver une architecture purement sensorielle ${ }^{1}$ ". On sait cependant que, bien plus que l'architecture, la peinture ou le cinéma lui fournissent références et schèmes d'écriture. Occupant une place secondaire dans l'œuvre, l'architecture figure néanmoins en bonne place parmi les expériences et les paysages qui composent sa mémoire. Sa présence est souvent liée à la question de la modernité, et les liens alors tissés entre les deux notions retiennent l'attention: la littérature, depuis la fin du XIX ${ }^{e}$ siècle, nous a en effet habitués à associer architecture urbaine et modernité, une modernité porteuse de valeur esthétique, dans la lignée de Baudelaire et d'Apollinaire.

Or l'architecture moderne dans le roman simonien fait l'objet de descriptions le plus souvent caustiques. S'intéresser à l'architecture consiste, pour l'oncle Charles dans Histoire, à décrire l'appareil du mur qu'il a sous les yeux: quand le jeune Lambert lui demande en quoi est construite " cette bicoque ", l'imposante et vénérable maison familiale, l'oncle lui montre ce mur fait d'" une rangée de briques et [d']une rangée de galets alternés ", "galets inclinés une fois dans un sens une fois dans l'autre c'est ce qu'on appelle l'appareil

1. Interview avec C. Sarraute, Le Monde, 8 octobre 1960. 
en arêtes de poiss ${ }^{2}$ ". Lambert lui coupe la parole pour affirmer, péremptoire "Le matériau de l'avenir c'est le ciment armé ", et l'oncle Charles juge inutile de répliquer. Dans cet affrontement de deux générations et de deux conceptions architecturales, du fait des personnages qui les incarnent fugitivement, la valeur est clairement du côté de l'ancien plutôt que du moderne. Ce peut être un point de départ pour interroger la relation de Simon à l'architecture. Sa poétique refusant tout mimétisme, toute description passe par l'expérience d'un regard, la description architecturale comme une autre: nous observerons d'abord la manière dont celle-ci s'anime, puis l'axiologie qui la fonde et les critères qui permettent d'affiner l'opposition majeure ancien/moderne.

\section{UN CEIL SUR LA VILLE}

Les objets architecturaux urbains apparaissent dans le roman simonien toujours pris dans le regard d'un personnage ou du narrateur, vus sous un certain angle. Un regard qui les ébranle, les dote de mouvement: la description se morcelle en instantanés successifs et figés, puisque tout mouvement ou toute action ne peut "se reconstituer" que "sous forme d'une série images fixes, figées, immobiles" $(P, \mathrm{p} .66)$.

Le regard posé sur la ville n'est que rarement le support d'une lecture sociologique telle que celle des villas fin de siècle qui bordent la fin du trajet du tramway (Tram., p. 15). C'est toujours un regard mobile, mouvement du spectateur ou du regard lui-même: pour l'étudiant du Palace emporté par une voiture folle à travers la ville nocturne, "déserte et vide " comme si elle avait été « coulée d'un bloc [...] dans une sorte de lave » $(P, \mathrm{p} .80)$, les monuments, maisons et églises incendiées se muent alors en autant d'obstacles en mouvement, de projectiles: «Puis il entendit hurler les pneus et [...] il vit quelque chose fait de pierre, de marbre et de métal entremêlés de façon compliquée et qui leur arrivait dessus à la vitesse d'une locomotive, femmes nues, tritons, rostres de navire, corniches, tout mêlé ${ }^{3}$ " $(P$, p. 81). Décrire le monument, c'est le comparer, énumérer les détails entrevus et, évitant de le nommer, le donner à percevoir et non à identifier. La description encyclopédique de la notice sur Barcelone, juxtaposée et insérée dans ce récit de la course de nuit à

2. Hist., p. 218. Les références aux romans de Claude Simon seront désormais données dans le corps du texte, à la suite des citations et selon le code adopté par les Cabiers Claude Simon.

3. Autre exemple similaire: "une apparition, un instant, aussi furieusement emportée dans le néant qu'apparue: quelque chose d'ouvragé, paradoxal, futile: le mur aveugle et précieux aux pierres taillées en pointes de diamant, les deux niches symétriques des saints décapités... ", P, p. 88. 
travers la ville ( $P$, p. 88-89), propose à l'inverse une vision synthétique, image ou texte ${ }^{4}$, et figée: elle ne relève pas de la sensation.

Le lent et pénible parcours du personnage des Corps conducteurs dans une rue new-yorkaise donne de la base des buildings qui bordent cette "tranchée ", une vision au rythme irrégulier de sa marche, et décrit des vitrines devant lesquelles s'arrête l'homme malade, ou les passants qui le croisent, pendant qu'il reprend quelques forces. C'est sa propre sensation de vertige qu'il transfère alors à l'architecture:

Depuis qu'il est assis, les hauts buildings ont cessé de dériver. D'un seul jet, ou parfois constitués de cubes de tailles décroissantes posés les uns sur les autres comme les marches de pyramides géantes, ils dressent dans la brume décolorée leurs entassements géométriques. (CC, p. 189)

Cette phénoménologie de la perception de l'espace urbain le décrit tel que ressenti et mis en mouvement par le déplacement du regard. La seule variation du point de vue suffit parfois à dynamiser l'architecture urbaine et plus particulièrement quand alternent point de vue rapproché et point de vue surplombant $^{5}$ : le sujet déplace son regard tantôt vers le proche et le détail, tantôt vers le lointain et la vision globale. Celle-ci n'offre pas de spectacle à déchiffrer, selon des oppositions sociologiques et des points saillants comme le panorama du XIX ${ }^{\mathrm{e}}$ siècle, même si cette dimension n'est pas absente du Tramway ou des Corps conducteurs, au second $\operatorname{plan}^{6}$. Le plus souvent, percevoir « la ville toute entière ", formule récurrente dans Le Palace, permet d'exprimer une impression globale, olfactive - la puanteur - plutôt que visuelle ${ }^{7}$. La perception est subjective, sans être celle d'un individu particulier, mais plutôt d'un anonyme ou d'un personnage pluriel: aussi bien l'étudiant que l'Américain, lui qui le premier compare la ville à une femme en train d'avorter $(P, \mathrm{p} .16)$. La seule vue panoramique de la Barcelone du Palace est une lecture par l'étudiant du plan affiché dans l'hôtel et biaisée par son expérience de révolution avortée:

et il semblait à l'étudiant la voir toute entière, d'un jaune sale, au bord de sa mer d'un bleu sale, décoloré, baignant dans cette espèce de brume blanchâtre [...]

4. Dans Le Jardin des Plantes, une vision globale et cependant mouvante de Moscou est donnée quand le narrateur sort de la ville en voiture: "Moscou se termine brusquement. En me retournant, je pouvais voir s'éloigner les lumières des hauts immeubles neufs qui la ceinturent. Comme une muraille ", JP, p. 50-51. Ce pourrait être une vue d'avion, comme il s'en trouve de nombreuses dans le roman.

5. Comme l'ont montré Michel Deguy, "Claude Simon et la représentation ", Critique, 187, déc. 1962, et Jean-Yves Laurichesse, "Aux quatre coins du monde. Le Jardin des Plantes comme album d'un voyageur " "Le Jardin des Plantes de Claude Simon », Cahiers de l'Université de Perpignan n 30, PU Perpignan, 2000.

6. Voir Claude Simon géographe, textes réunis par Jean-Yves Laurichesse, avec des photographies de Pascal Mougin, Classiques Garnier, 2013, 267 p.

7. « jusqu’à ce qu'ouvrant la fenêtre on se rappelât que ce n'était pas l'hôtel [...] qui puait ainsi, mais la ville toute entière, comme si elle était en train de se putréfier... », $P$, p. 14. 
les mornes et lourdes successions d'immeubles uniformément recouverts de cette crasse jaunâtre... (P, p. 17)

À l'opposé, l'aquatinte de Barcelone, dans l'atelier de l'oncle Charles, longuement décrite, est une parodie de panorama, une image-modèle, avec une " rue modèle " et des quartiers modèles: " on aurait dit un de ces tableaux muets, éducatifs et microcosmiques suspendus autrefois aux murs des classes enfantines " (Hist., p. 164). C'est en opposition avec cette vue générale et figée que vient s'inscrire - en réponse à la question "Comment était-ce? », le détail grossi du mur contre lequel s'était collé le narrateur: " presque aussitôt le bruit saccadé encore je m'aplatis un peu plus contre le/ mur ou plutôt soubassement appareil de pierres jaunâtres faisant saillies c'est-à-dire chaque pierre rectangulaire taillée de la façon suivante:..." (Hist., p. 176).

Ce va-et-vient entre plan d'ensemble et gros plan devient alternance entre vue plongeante et contre-plongée dans la description de la rue new-yorkaise des Corps conducteurs: d'abord prêtée à l'homme malade qui a dû s'arrêter, la description, partant du détail de l'appareil du mur «constitué de larges blocs de pierre grise jusqu’à hauteur d'homme " passe par « la façade qui, au dessus, est en briques brunâtres ", pour finir sur:

les lignes de fuite convergentes interrompues à la hauteur du vingtième étage et que l'œil prolonge vers leur point de rencontre dans le vide éblouissant et décoloré. Pris d'un léger vertige, il abaisse la tête, son regard parcourant maintenant de haut en bas la façade brune puis grise. (CC, p. 13)

Du tout proche au lointain puis à nouveau au tout proche, le regard vacille. Plus loin, ce parcours s'inverse, partant " des plus hauts étages des gratte-ciel » d'où « ils semblent flotter, suspendus, verticaux, géométriques et sans poids, sur une base immatérielle, comme les sommets des montagnes dans les lavis des peintres chinois" et là encore débouche sur le vide (CC, p. 23). Or ce regard impersonnel (" on », « l'œil ») qui renverse la perspective, efface ce qui est à la portée du regard de l'homme malade: depuis ce point de vue élevé, " il est impossible de distinguer le trafic et la foule qui s'écoulent au fond des canyons de pierre " (ibid.). En fait, le lecteur comprend vite que le regard s'est fixé sur une photographie de gratte-ciel dans une vitrine. Va-et-vient et basculement disent un vertige qui est ici transmis au lecteur pour quelques secondes d'incertitude sur la représentation. "Proche, trop proche, éloigné, trop éloigné, le regard va et vient, le regard est l'incessant va-et-vient de l'impossible accommodation $^{8}$ " écrivait Michel Deguy en 1962.

Seule peut-être, la vue d'avion permet, sans vertige, une liberté d'accommodation qui permet de saisir un détail du paysage urbain tout en l'embras- 
sant dans son ensemble: ainsi la vue de Chicago du voyageur du Jardin des Plantes isole deux éléments, le lac " comme une plaque d'or " et un gratte-ciel:

Près de sa rive, et dominant tous les autres, un gratte-ciel s'élevait, non pas parallélépipède comme ses voisins mais semblable, en gigantesque, à l'un de ces échafaudages (derricks?) dressés au-dessus des puits de pétrole, c'est-à-dire en forme de pyramide étirée et tronquée. De l'une à l'autre de ses arêtes, s'entrecroisaient en oblique des poutrelles de fer. Il était entièrement peint en noir. L'or du lac semblait comme en fusion: Chicago ${ }^{9}$. (JP, p. 53)

Il y a du bonheur dans cette double accommodation. Un autre plaisir de la vue d'avion est de décrire une ville en cours d'apparition comme cette ville soviétique de L'Invitation, qui ne s'annonce pas par " ces éparpillements de lumière, qui entourent habituellement les villes " mais comme " un bloc, c'est-à-dire une tache nette, fermée, close pour ainsi dire sur elle-même» (I, p. 28).

Enfin, l'écriture peut, par ses seuls pouvoirs, insuffler le mouvement à l'architecture, par le jeu des métaphores, comparaisons ou antithèses qui dynamisent la description architecturale. Les métaphores transforment les constructions en éléments naturels: l'avenue new-yorkaise devient " canyon " entre des montagnes et le flot des voitures, "rivière "; les buildings sont des "falaises " $(C C$, p. 22). Ces mêmes métaphores décrivent Barcelone en proie à la violence, et qui, dépouillée de son apparence quotidienne, devient espace sauvage ${ }^{10}$ (Hist, p. 185). Les métaphores animales et humaines disent l'architecture baroque ${ }^{11}$ de la ville: son palace et la masse organique qui l'entoure, puante "comme si elle était en train de se putréfier» ( $P$., p. 14), une sorte de géante qui "même la nuit, continuait à suer, suinter " et enfante dans la douleur le foetus mort-né de la révolution. Ou encore, sous le regard de l'étudiant tourmenté par la disparition inexpliquée de l'Américain, la façade menaçante du palace:

à la fin, il se recula, levant la tête vers la haute falaise de pierre, qui se dressait au-dessus de lui avec ses entablements, ses consoles, ses balcons, ses redans, comme la concrétisation furieuse de quelque délire [...] si bien qu'au bout d'un moment ceux-ci semblèrent basculer, comme si la façade toute entière s'inclinait, se rabattait en avant [...] elle les écraserait tous sous un apocalyptique « entassement... » $(P$, p. 189-90)

Matière d'expériences sensibles bien plutôt qu'objets d'architecture urbaine, la ville est à la fois le lieu et le partenaire; et parce que l'écriture tente de resti-

9. Dans ce dernier livre particulièrement, sont fréquentes les vues plongeantes sur des villes, toutes différentes, et toutes nommées, contrairement à la plupart des livres antérieurs.

10. «Chose qui avait été autrefois simplement une avenue avec des passants des bonnes poussant des landaus sous les ombrages des voitures [...] et maintenant rien que deux falaises de pierre jaunâtres plates mornes se faisant vis-à-vis et dans le fond desséché du canyon cette voiture pareille à un rhinocéros foudroyé en pleine charge ", Hist, p. 185.

11. Ainsi, « la fastueuse débauche de corniches, de volutes et de vagues pétrifiées détournée de sa destination première ", $P$, p. 14. 
tuer l'expérience perceptive que sont les villes, leurs différences architecturales s'effacent dans des métaphores analogues, signifiant le malaise, le vertige, ou la menace. L'opposition entre Barcelone et New York se voit minimisée par une même phénoménologie littéraire, qui restitue une expérience de basculement de la réalité et de bouleversement du regard, de retour forcé aux perceptions premières, et au détail, détail d'un appareil mural ou d'une vitrine à la base d'un gratte-ciel.

\section{ARCHITECTURE ET VALEUR}

La question de la valeur est implicitement posée dans la plupart des descriptions architecturales de Simon et se fonde le plus souvent sur une axiologie qui oppose architectures moderne et ancienne. Ainsi, la ville du Tramway est décrite comme la répudiation de la ville ancienne par la ville moderne: "une sorte d'antithèse d'elle-même sous les aspects d'une modernité d'ailleurs presque aussitôt fanée » (Tram., p. 79). Ce sont les formulations de cette antithèse que je voudrais d'abord explorer.

\section{L'ancien et le moderne}

Sur les architectures modernes, les jugements négatifs ne manquent pas. Ainsi l'évolution de Perpignan, jamais nommée, est décrite comme un reniement de ses propres valeurs quand l'embellissement se fait par l'importation d'"un Crystal palace en réduction" (Tram., p. 109) puis comme un faux retour à l'authenticité motivé par des raisons commerciales et touristiques. De même, les quartiers modernes de Barcelone décrits à partir de l'aquatinte et du souvenir, sont des "quartiers modèles, jaunes, neufs, florissants et lugubres " (Hist., p. 167). L'ironie à l'égard des constructions récentes est récurrente et moque l'emphase et la prétention esthétique, mais également la fausse modernité du progrès industriel et technique des:

éléphantesques monuments élevés à la gloire de la Production Industrielle et du dieu Commerce dans une combinaison des divers ordres architecturaux grecs florentins victoriens et des derniers progrès de la technique en matière d'armatures métalliques, d'ascenseurs, de verrières et de pilastres de fonte (Hist., p. 161)

L'ironie porte aussi sur le mode de construction standardisé et industrialisé des zones périphériques: les abords de Perpignan où revient le narrateur d'Histoire sont faits de « cubes gigantesques blafards apparemment démoulés d'un coup cuisine et formica compris facétieusement déposés au milieu d'étendues marron défoncées ${ }^{12} "$ (Hist., p. 326). Ce sont sans doute les villes soviétiques de

12. Autres exemples: la description des immeubles de Perpignan dans le crépuscule les montre «s'élevant sur le sol nu comme à la surface de quelque planète perdue morte avec leurs cargaisons d'hommes et de 
L'Invitation, comme Frounze au Kirghizistan, « surgie de la steppe » (I, p. 35), qui portent à son comble le mode de construction de la ville moderne érigée d'un seul bloc. Simon en décrit les "fastueuses architectures" ( $I$, p. 36) et oppose à la cité "lentement constituée au cours du temps, par bourgeonnements successifs, à partir d'un carrefour de routes, d'un marché [...] », cette ville " comme surgi[e] tout à coup du néant... ou d'un passé $[\ldots]$ néantisé à coups de bulldozers pour édifier à la place ce que les architectes diplômés des écoles d'architecture et les urbanistes diplômés des écoles d'urbanisme appellent aujourd'hui une ville" (I., p. 58). C'est leur genèse qui fait de certaines agglomérations modernes des villes coupées de leur passé, de l'histoire, entièrement circonscrites et réduites au présent.

Le jeu de l'antithèse valorise ainsi l'architecture ancienne, même si celle-ci est rarement évoquée positivement. Ainsi la maison, ou le "mausolée " familial, forme une sorte d'îlot silencieux au milieu de la cité envahie de néons et de bruit, et s'oppose aux froides salles de marbre de la banque. Sa construction ancienne est colorée d'affect et la mère du narrateur est comparée à « l'un de ces hauts murs nus bordant une rue, impénétrables, hautains, secrets ", défendue "par rien d'autre que par une formidable inamovibilité » (Hist., p. 20). C'est de la même manière différentielle que la juxtaposition des évocations de la Rome ancienne et moderne, la première faite par le narrateur du Jardin des Plantes, la seconde par son ami italien, Gastone Novelli, induit un jugement de valeur positif porté sur la Rome ancienne, confirmé par la métaphore des ossements que se disputent des prédateurs avides.

Avec ses ruines colossales, sa profusion de palais, de coupoles et d'églises entre lesquels circulait (ou se trouvait coincé) le flot des voitures, ça faisait penser aux ossements de quelque monstre prédateur d'une espèce disparue depuis longtemps et dont une armée d'insectes à carapace s'acharnait à ronger ce qui pouvait encore rester de chair accrochée à ces falaises de pierres, ces arcades, ces thermes, ces dômes boursouflés et creux. (JP, p. 118)

Questionné sur la Rome actuelle, l'Italien répond que "c'était toujours le même genre de bandits ", " construisant des merdes en ciment armé faites tout au plus pour durer une vingtaine d'années et tout juste bonnes ensuite à dynamiter» (ibid., p. 119).

Et pourtant, il y a moderne et moderne, et Claude Simon aime les gratteciel de New-York. Parlant de sa découverte de la ville en octobre $1968^{13}$, il dit

femmes mangeant dormant et se reproduisant par rangées superposées ", Hist. p. 326; la ville neuve de Göteborg dans Le Jardin des Plantes qui «ne présente aujourd'hui, avec sa large avenue centrale bordée de bâtiments modernes sans caractère, qu'un médiocre intérêt, à l'exception d'un important chantier naval... ", JP, p. 358.

13. «J'aime la modernité, je n'aime pas le modernisme [...] j’aime les gratte-ciel de New York, je n'aime pas le Forum des Halles "; propos tenu en 1990, cité par Mireille Calle-Gruber, Claude Simon, Une vie à écrire, Le Seuil, 2011, p. 418. 
avoir ressenti un « choc physique devant cette verticalité de la ville, ces plans, ces cubes, ce rythme extraordinairement net et heurté. Il s'est agi d'un événement aussi important pour ma sensibilité que certains faits de mon passé liés à la guerre ${ }^{14}$ ".

L'expansion de la ville provinciale d'Histoire et du Tramway avec ses immeubles de "ciment armé " autrement dit de béton, est décrite en termes d'explosion horizontale (Tram., p. 79). Tout autre est l'explosion verticale des métropoles américaines, comme celle de New York dans Le Jardin des Plantes et Les Corps Conducteurs. L'évocation de celle-ci porte à son degré extrême la dynamique descriptive déjà notée: la métaphore de l'explosion revient mais verticale et valorisée: "non pas exactement debout, statique, mais explosant, toujours en expansion non pas en surface mais en hauteur ». Le mouvement ascensionnel s'accompagne d'un emboîtement étonnant de comparaisons et métaphores: il est d'abord question de

certaines photographies prises d'avion [...] avec un objectif grand angle [...] exagérant la perspective, de sorte que ses multiples gratte-ciel apparaissent non pas verticaux, parallèles, mais obéissant à une force divergente, faisant penser à ces gerbes de cristaux allant s'écartant, se bousculant, poussant vers le ciel ses tours de toutes hauteurs, les moins élevées non pas résignées $[\ldots]$ mais $[\ldots]$ se dépêchant pour rattraper les autres.

La comparaison technique en engendre une autre avec un phénomène naturel (les "gerbes de cristal»), puis génère une personnification, et puis encore, dans un formidable effet de recul de la perspective, une comparaison cosmique ( l'ensemble comme planté sur la rotondité bombée de la terre...», $J P$, p. 284). Quand la métaphore de l'explosion est reprise en fin de paragraphe par "une sorte d'explosion solidifiée " (qui peut rappeler la redéfinition surréaliste de la beauté " explosante-fixe »), il me semble difficile de ne pas voir là un jugement esthétique. La valeur esthétique est confirmée par la chute du paragraphe descriptif: "(une sorte) de phénomène naturel, anarchique, tumultueux et géométrique ». Cette architecture qui allie les contraires, le naturel et le géométrique, l'ordre et désordre, apparaît comme la forme de modernité architecturale créatrice, harmonieuse et oxymorique.

Dans le montage qui fait alterner les séries que Le Jardin des Plantes dispose en damiers, la description de New York se distingue en ce qu'elle n'est pas isolée mais juxtaposée et en contraste avec celle de Saint-Pétersbourg. C'est le rapport au temps et au pouvoir politique qui différencie les deux villes, cette dernière décrite comme

soudain posée horizontale, d'emblée, entièrement dessinée à l'avance, à plat, par le même architecte, jusqu'au moindre de ses ornements rococo, ses entablements, ses atlantes [...], et là une fois pour toutes. Édifiée à bras (et à mort) d'homme sur un marécage plat. (JP, p. 284)

14. Tribune de Genève du 10 juin 1970, cité par Mireille Calle-Gruber, ibid., p. 304. 
Son caractère monolithique, son horizontalité, son absence d'évolution en font une antithèse de New York, et son lien avec le pouvoir autocratique de Pierre le Grand est établi par la description juxtaposée du " terrifiant » mannequin de cire du tsar au musée de l'Ermitage ( $J P$, p. 285). Une ville terrifiante aussi sans doute pour Simon. La contiguïté des deux descriptions souligne leur opposition dans l'axiologie des valeurs architecturales, et celle-ci ne se fonde donc plus seulement sur le critère d'ancienneté, puisqu'elle valorise certaines architectures modernes et dévalorise certaines villes anciennes. Un autre critère intervient, la relation au pouvoir politique et le mode d'édification de la ville - l'architecture de Saint-Pétersbourg étant entièrement déterminée par un pouvoir autocratique; et les villes soviétiques modernes, on l'a vu, relèvent du même urbanisme planifié par un pouvoir fort. Moderne ou ancienne, une ville doit s'être érigée dans et avec le temps.

\section{LE MUR ET LE TEMPS}

Un autre critère d'évaluation architecturale apparaît dans Histoire, les matériaux de construction, auquel Simon prête une attention extrême, et ce n'est pas seulement parce qu'il y voit une métaphore scripturale ${ }^{15}$. Ce qui le retient, c'est le faire du constructeur, le matériau de construction et le geste de l'artisan, comme le travail de l'architecte qui le guide.

\section{La brique, le galet et le ciment armé}

Dans la discussion de l'oncle Charles et de Lambert évoquée en ouverture, la différence des matériaux, ici galets ou briques, là ciment ou béton armé, valait pour une opposition entre l'ancien et le moderne, et induisait un jugement négatif sur l'architecture moderne: la phrase de Lambert, présenté plus loin comme un "agressif et pitoyable coq" (Hist., p. 326), condamne implicitement son contenu: le ciment armé est sans valeur face à l'appareil du vénérable mur de briques et de galets. De surcroît, la discussion fait écho à une page où la métaphore du ciment - matériau de base du béton armé - figurait le figement et la mort: il s'agit d'une carte postale du port d'Aden, "non pas un port $[. .$.$] mais un ciel de ciment, des flots de ciment » et un « quai absolument$ désert », " comme si les hommes, après l'avoir imaginé, dessiné puis construit, planté dessus réverbères et hangar, s'étaient ensuite enfuis épouvantés, tout retombant, après l'assourdissant tapage des bétonneuses et des ordres criés, dans le silence originel»(Hist., p. 58). Le ciment et le béton, matériaux de

15. On pense à la photographie de mur de galets que C. Simon intitule "Page d'écriture ». Voir les deux pages que consacre Lucien Dällenbach aux matériaux et à l'imaginaire du mur dans son Claude Simon, coll. « Les contemporains », Le Seuil, 1988, p. 124-126. 
base de l'architecture industrielle, préfabriquée et inhumaine, produisent des espaces mortifères pour la nature même aux alentours ${ }^{16}$. Or l'affirmation de Lambert sur le béton armé comme " matériau de l'avenir ", quoiqu'arrogante dans son contexte fictionnel et lourde d'un mépris ridicule pour l'architecture ancienne, n'en reste pas moins fort juste. Ce jugement d'un adolescent épris de modernisme et qui reproduit sans doute un propos d'adulte, manifeste une lucidité certaine sur " les possibilités infinies rationnelles» (Hist., p. 218) d'un matériau (le ciment armé ou le béton) sur lequel porte les recherches des architectes de la modernité (Tony Garnier, Auguste Perret et surtout Le Corbusier ${ }^{17}$ ) et qui se révélera - plus encore après la fin de la guerre - matériau fondateur de toute l'architecture moderne, pour le meilleur et pour le pire.

Sans doute le critère esthétique est-il ici déterminant dans l'opposition entre mornes immeubles modernes et gratte-ciel des villes américaines. Cependant le matériau n'en reste pas moins essentiel au style architectural. De l'attention pour le matériau en lui-même témoigne encore cette notation de détail de la scène de la mort du colonel où sont différenciées les briques anciennes des briques mécaniques:

Briques des murs: non pas lisses comme ces briques mécaniques qu'on fait aujourd'hui mais grenues, rêches: brun rouge, brun violacé, certaines presque bleues, d'un bleu gris, ferrugineux. Arrachées du fond de la terre. Pays minier pas loin. (JP, p. 311).

Le matériau ancien est expressif et, à la différence du matériau industriel, dit l'ancrage dans le sol du territoire, comme les couleurs de la brique indiquent un sol ferrugineux, ou les galets la pierre du sud-ouest.

Même si le béton armé autorise la construction en série des habitations uniformes de l'architecture industrielle, il est aussi le matériau qui libère l'architecte des contraintes antérieures et lui permet, avec le plateau libre, les colonnes porteuses, les façades libérées et la plasticité des formes, la créativité qui sera précisément celle des premiers gratte-ciel de Manhattan ou des premières constructions du Corbusier ou de Niemeyer.

16. Plus loin, la vision des «cubes gigantesques» des abords de la ville fait resurgir chez le narrateur le souvenir du " ciment armé " vanté par Lambert " disant que ça au moins c'était moderne pas comme ces ", Hist., p. 326. Récurrent dans Histoire, le jugement sévère sur le mode de production industriel d'immeubles et de villes entières revient dans l'Invitation, lorsque les villes modernes d'URSS, sont décrites comme sorties de "l'unique et gigantesque usine qui produisait en série les villes conçues par ordinateur ", $I$, p. 63.

17. Voir par exemple les écrits d'Auguste Perret, Anthologie des écrits, conférences, entretiens (Laurent C. \& Lambert G. \& Abram), Édition Le Moniteur, 2011, 480 p.; et parmi ceux du Corbusier, son Entretien avec les étudiants des écoles d'architecture, Minuit, 1958 ou Vers une architecture (1923), Flammarion, 2008. 


\section{Fascination pour les ruines}

Cependant, la méfiance à l'égard du béton armé tient sans doute à ce que pour Simon tous les matériaux n'offrent pas la même résistance au temps, c'est-à-dire à la destruction. On se souvient de la modernité " presque aussitôt fanée " des immeubles du Tramway (Tram., p. 79) ou de ceux de la Rome actuelle (JP, p. 119). Il faut les rapprocher des "petites agglomérations en ruines que l'on appelle les Ghost Cities " $(J P$, p. 69) près des Montagnes rocheuses pour éclairer le lien qu'établit Simon entre la résistance aux agressions du temps et les matériaux utilisés:

Contrairement $[\ldots]$ aux ruines laissées derrière elles par les civilisations passées et dont on peut voir encore les vestiges (temples, forums, remparts, arènes), les matériaux employés là (le bois et le fer), de nature périssable, sont condamnés à être peu à peu attaqués et détruits [...] de sorte qu'à la différence des sanctuaires, des colonnades ou des agoras, il ne restera un jour absolument rien, [...] de ce qu'un furieux et hâtif appétit de gain a édifié, le sol de nouveau aride, à peine bosselé encore sur quelques décombres, puis simplement nu, retourné à son état primitif. $(J P, \mathrm{p} .70)$

L'opposition entre les architectures moderne et ancienne tient donc aussi à la capacité des secondes à résister au temps: la dégradation des cités anciennes ne produit pas un néant mais des ruines et des vestiges, c'est-à-dire encore de la beauté, de la force, et un support pour l'imaginaire; certaines architectures urbaines, en revanche, ne sont destinées à rien d'autre qu'à la mort. C'est donc aussi en fonction de leur devenir et de ce qu'elles laisseront derrière elles que les architectures et les cités modernes sont condamnées: non pas seulement quand elles n'intègrent pas leur passé, mais quand ou parce qu'elles n'ont pas de futur, parce que leur devenir ruines est menacé. La ruine est vestige pour Simon, trace laissée par le passé, et, par là même, dotée d'une valeur que l'édifice neuf n'avait peut-être pas:

Après tout, les ruines sont des manifestations de la vie dans ce qu'elle a de plus robuste, et tout passé est une addition de ruines auxquelles le temps, les mutilations, confèrent une majesté durable que l'édifice ainsi ennobli n'avait pas à l'état neuf. Nous sommes tous constitués de ruines: celles des civilisations passées, celles des événements de notre vie dont il ne subsiste dans notre mémoire que des fragments ${ }^{18}$.

Les ruines antiques constituent le modèle même des ruines majestueuses: du Berlin des années 1960, dans Le Jardin des Plantes, seules sont décrites les ruines du centre-ville: " un silence total, insolite, enveloppe les murailles calcinées et les amoncellements de pierres et de briques ", des ruines envahies de végétation comme des "ruines antiques" (JP, p. 162). Certes la vision d'une Barcelone en décomposition dans Le Palace, une ville faite de décombres, ne donne guère une image positive de la ruine. Il en est tout autrement des ves- 
tiges grecs ou égyptiens, comme le « corridor de murailles cyclopéennes » du tombeau royal géant visité en Grèce (Hist., p. 103), ou comme les ruines du temple de Médinet-Abou (JP, p. 362) près des colosses de Memnon « monumentaux, insolites et solitaires ": "sur les hautes murailles qui en encadrent l'entrée, les vents et les sables ont aussi rongé, presque effacé, les légers bas-reliefs où l'on déchiffre à peine les formes de chevaux..." (ibid.) Dans les deux cas, le narrateur évoque une activité imaginaire: ici reconstitution de bas-relief effacés, et plus haut, dans Histoire, recherche "sous les décombres, les blocs effondrés, les débris ", de "ce qui avait été, ce qui..." (Hist., p. 103). Si les ruines sont des manifestations de la vie, c'est aussi parce qu'elles incitent à un travail de l'imagination, comparable au travail élaboré par l'écrivain à partir des fragments de sa propre mémoire.

Sans doute la fascination pour les ruines tient-elle enfin aussi à ce qu'elles soulignent la fragilité des constructions humaines, comme celle de la verticalité des édifices et des hommes. Le narrateur d'Histoire note, à propos de décombres, la faculté des objets à

se muer par simple rotation de cent quatre-vingts degrés autour d'un axe horizontal en autant d'épaves emphatiques et terrifiantes comme pour rappeler qu'à tout instant le monde ordonné et rassurant peut soudain chavirer, se retourner et se mettre sur le dos [...] et retournant au chaos originel, en dévoiler la face cachée... (Hist., p. 66)

La ruine est avertissement, mise en garde et incitation à vivre. Elle est ainsi liée à la notion de "mélancolie » telle que l'entend Simon, non pas la notion mièvre que l'on entend par là en général, mais " quelque chose de violent qui protestait, furieux, bâillonné mais hurlant ", "cette déchirante et mélancolique avidité avec laquelle le condamné regarde autour de lui le monde " $(J P$, p. 303, 305).

L'architecture et singulièrement l'architecture urbaine est ainsi prise dans une expérience sensible, mais aussi jugée, évaluée en termes esthétiques, politiques, éthiques. Elle pose à l'écrivain la question brûlante de la modernité et de son paradoxe: comment éviter que la modernité ne soit qu' " une modernité d'ailleurs presque aussitôt fanée "? On comprend que la question importe, et sans doute faut-il voir là l'origine de l'importance des matériaux dans sa réflexion sur l'architecture: le matériau qui ne disparait pas totalement et qui se prête à la recherche et à la reconstitution de traces, pour l'écrivain, qu'est-ce d'autre que l'écriture? 